\title{
Multi-level Interventions to Promote Oral Pre-exposure Prophylaxis Use Among Adolescent Girls and Young Women: a Review of Recent Research
}

\author{
Elizabeth Irungu ${ }^{1} \cdot$ Nomhle Khoza $^{2} \cdot$ Jennifer Velloza ${ }^{3}$ (I) \\ Accepted: 26 August 2021 / Published online: 31 October 2021 \\ (c) The Author(s), under exclusive licence to Springer Science+Business Media, LLC, part of Springer Nature 2021
}

\begin{abstract}
Purpose of review This review summarizes interventions to promote HIV pre-exposure prophylaxis (PrEP) use among adolescent girls and young women (AGYW) in HIV endemic settings, while also highlighting gaps in our current measures of PrEP intervention success.

Recent findings AGYW report challenges with PrEP use, although the field is currently grappling with defining metrics of optimal PrEP use applicable for AGYW with dynamic HIV prevention needs. Ongoing studies are exploring multilevel interventions to address barriers to PrEP use for AGYW. At the individual and interpersonal levels, mHealth, drug-level feedback, adherence counseling, peer groups, and PrEP decision-support interventions are acceptable and feasible for AGYW although limited effectiveness data are available. At the health facility and community levels, PrEP demand creation, modified PrEP refill schedules, and integrated PrEP and reproductive health services are also promising options to support PrEP use for AGYW. Summary As PrEP delivery continues to expand, improved metrics of success and evidence on the effectiveness of multilevel adherence support interventions are needed to maximize the impact of PrEP for AGYW in HIV endemic settings. We present case studies of these intervention approaches but limited data are currently available on the effectiveness of these approaches. We will look toward forthcoming study results on the impact of PrEP interventions, including mHealth, druglevel feedback and other enhanced counseling, peer support, decision-support tools, PrEP demand creation, modified refills, and integrated service delivery, to determine the ideal package of PrEP support approaches for AGYW.
\end{abstract}

Keywords Pre-exposure prophylaxis · Adherence $\cdot$ Uptake $\cdot$ Prevention-effective use $\cdot$ Adolescent girls and young women . HIV prevention

\section{Introduction}

Adolescent girls and young women (AGYW), ages 16-25 years, face alarmingly high rates of HIV, with recent trials reporting HIV incidence estimates as high as 7 per 100

This article is part of the Topical Collection The Science of Prevention

Jennifer Velloza

jvelloza@uw.edu

Partners in Health and Research Development, Thika, Kenya

2 Faculty of Health Sciences, Wits Reproductive Health \& HIV Institute (Wits RHI), University of the Witwatersrand, Johannesburg, South Africa

3 Department of Global Health, University of Washington, 325 Ninth Avenue, Seattle, WA 98104, USA person-years in this group [1, 2•,3]. As a result, AGYW are a priority population for effective HIV prevention options, including oral pre-exposure prophylaxis (PrEP). The World Health Organization recommends oral PrEP for AGYW and approximately 120 countries have included PrEP recommendations in their national guidelines [4]. Randomized controlled trials found that oral PrEP is $>90 \%$ effective at preventing HIV when taken with high adherence during periods of risk [5-8]. Demonstration projects with adolescents and young adults report high initial uptake of PrEP in real-world delivery settings $[9,10,11 \bullet \bullet, 12,13]$. However, despite its effectiveness, some AGYW have difficulty adhering to PrEP and sustaining continued PrEP use, limiting its impact as a biomedical HIV prevention tool [14•].

Recent PrEP programs with AGYW report declining adherence over the first 6 months to 1 year of use, due to a range of individual, interpersonal, and contextual factors 
$[11 \bullet \bullet, 12,13,14 \bullet, 15 \bullet \bullet, 16]$. Commonly reported barriers to PrEP use in this population include lack of social support, stigma and discrimination related to sexual behavior and PrEP use, gender-based violence (GBV), PrEP side effects, forgetting pill dosing and lack of habit formation around use, disclosure concerns, and depressive symptoms [13, 17-20]. Judgmental or stigmatizing interactions with health care staff, fears of provider judgmental, long clinic wait times, and inconvenient clinic opening hours and visit schedules also may impact PrEP uptake and refills [13, 16, 21].

Successful PrEP delivery for AGYW will require a range of PrEP support interventions that address these complex, multi-level challenges to regular PrEP use during periods of HIV risk. This review will summarize current evidence on PrEP support interventions for AGYW, with a particular focus on recent interventions to address individual, interpersonal, and contextual barriers to PrEP use.

\section{Text of Review}

\section{Defining Metrics of PrEP Success to Improve PrEP Delivery Targets for AGYW}

PrEP programs are currently seeking to identify effective interventions to maximize PrEP use among AGYW in HIV endemic settings, but they are simultaneously grappling with definitions around what it means to achieve "successful" oral PrEP use for this population. PrEP outcome measures have closely resembled metrics for antiretroviral therapy (ART) adherence, which do not adequately reflect the intention for PrEP to be used only during periods of HIV risk rather than as a lifelong treatment approach [22••]. Going forward, there is a need to move toward evaluating PrEP support approaches based on whether they promote alignment between PrEP dosing and dynamic changes in sexual behavior and HIV prevention needs among AGYW [23]. In this section, we present different perspectives on existing metrics of "PrEP success" to inform our findings and conclusions about effective PrEP support interventions presented elsewhere in the review.

PrEP uptake is a key outcome for PrEP trials and delivery programs among AGYW [22••, 24, 25]. Uptake is typically measured by receipt of a PrEP pill bottle or PrEP prescription. At subsequent clinic visits, PrEP adherence is generally assessed using a variety of self-report, electronic, and pharmacologic measures, including validated adherence questionnaires, pill counts, electronic pill containers, and plasma, dried blood spot, plasma blood mononuclear cell, hair, and urine laboratory or point-of-care assays [26•, 27]. PrEP persistence and continuation are often defined by pill pick-ups, visit retention, and adherence metrics assessed over a sustained period of time. While there is a range of measurement options for each of these PrEP outcomes, the numerators and denominators of these metrics are poorly defined and often differ by setting and population, limiting their comparability [22••]. For example, AGYW are deemed eligible for PrEP if they are "at substantial risk of HIV", which is based on a range of factors including regional HIV incidence and prevalence estimates, individual sexual behaviors, alcohol or substance use, GBV victimization, demographic factors including age, and any incident sexually transmitted infections (STIs) [24, 28]. Metrics of PrEP adherence, persistence, and continuation often do not account for changing HIV risk and need for PrEP over time in populations of AGYW.

PrEP demonstration and implementation projects with AGYW are currently exploring new measures of success for programmatic PrEP delivery. A recently published "intervention-centric cascade" outlines guidance for metrics for: (1) PrEP coverage (which incorporates statistics for the target population of AGYW, PrEP availability, uptake of PrEP screening, oral PrEP eligibility based on local guidelines, and oral PrEP offering, along with PrEP initiation and continuation); (2) PrEP update (inclusive of statistics on both PrEP offering and PrEP initiation); and (3) PrEP adherence (inclusive of statistics on PrEP initiation, PrEP continuation, and PrEP discontinuation) [22••]. PrEP restarts are also an important component of programmatic PrEP delivery and are a key success metric for AGYW with changing PrEP needs. The DREAMS, POWER, and Partners Scale-Up PrEP programs provide important examples of approaches to operationalize these new metrics of PrEP delivery for

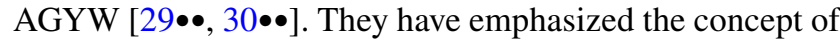
PrEP restarts to describe AGYW cycling on and off PrEP as a metric of success in this population.

Recent work has also been done to redefine metrics of PrEP adherence, recognizing that traditional measures of adherence that do not account for dynamic HIV prevention needs often fail to capture successful PrEP use for AGYW accurately. PrEP is most cost-effective when its use is aligned with periods of HIV risk - a concept defined as "prevention-effective adherence" [31•, 32]. Studies exploring prevention-effective adherence among AGYW have found that young women define their HIV risk based on various factors, including trust in a relationship and relationship dynamics, sexual behavior (e.g., number of sexual partners, transactional sex, condom use), pregnancy, and STI

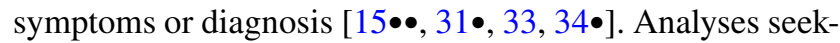
ing to understand prevention-effective PrEP use (by using a combination of information on sexual behavior, HIV risk perceptions, and biomarkers of sexual activity and PrEP use) have found that AGYW can align their PrEP use with peri-

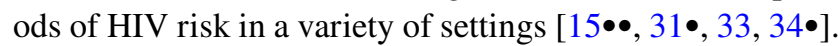
In addition, a cohort of AGYW using PrEP in South Africa and Zimbabwe reported low levels of PrEP continuation and 
PrEP adherence based on pharmacological samples but also found significantly lower than expected rates of HIV incidence $[15 \bullet \bullet]$. STI diagnoses were generally high (30-40\% of AGYW had STI diagnoses at enrollment) in this cohort, indicating some HIV risk, and supporting the effectiveness of PrEP dosing around periods of HIV risk to reduce incident HIV infections [15••]. PrEP programs could support prevention-effective PrEP use among AGYW by counseling clients to recognize drivers of HIV risk and modulate PrEP use accordingly. This body of research also points to a need for defining successful PrEP adherence based on a prevention-effectiveness model which may be effective for AGYW while also reducing cost, personnel, and time burdens on healthcare systems in HIV endemic settings.

\section{Individual and Interpersonal Interventions to Support PrEP Use for AGYW}

The context of PrEP measurement provides important background for our consideration of recent efforts to improve PrEP use among AGYW. Individual- and interpersonal-level interventions seek to address various barriers to PrEP use, including internalized, perceived, and experienced stigma around PrEP use, knowledge about PrEP pill taking, habit formation around PrEP dosing, and disclosure concerns. These interventions include: (1) mobile health ("mHealth") approaches; (2) counseling based on levels of PrEP detected in pharmacologic samples ("drug-level feedback counseling"); (3) counseling focused on these PrEP barriers using problem-solving, cognitive-behavioral, and motivational interviewing approaches; (4) peer support groups and adherence clubs; and (5) decision tools to facilitate decisionmaking around PrEP use (Table 1). The five intervention types seek to improve PrEP outcomes through increased PrEP knowledge, self-efficacy, behavioral skills for daily pill taking, social support, and youth-friendly communication between healthcare providers and clients. There is currently limited evidence on the effectiveness of these approaches on modifying PrEP uptake and adherence (defined as drug use over a period of time, rather than prevention-effective PrEP use) but we present a state of the current science and point to areas of ongoing research.

\section{mHealth interventions}

Several PrEP projects are currently assessing the use of mobile and wireless technologies such as text messaging, WhatsApp and other smartphone applications as a mechanism to support PrEP uptake, adherence and continuation while leveraging the high levels of mobile phone and social media use among AGYW. Currently, mHealth interventions to support PrEP use include one-way and two-way SMS messaging to remind AGYW to take PrEP and to facilitate real-time communication between health providers and

Table 1 Summary of current findings on individual- and interpersonal-level interventions to support effective PrEP use

\begin{tabular}{|c|c|c|c|}
\hline Intervention & Study & Key findings & Potential barriers to delivery \\
\hline \multirow[t]{4}{*}{ mHealth } & HPTN 082 & SMS were acceptable & SMS fatigue, smartphone access, phone data \\
\hline & MPYA & $\begin{array}{l}\text { SMS reminders were ineffective in promoting } \\
\text { PrEP adherence }\end{array}$ & \\
\hline & PrEP SMART & Ongoing study & \\
\hline & POWER & $\begin{array}{l}\text { SMS was motivating and helped participants } \\
\text { remember clinic visits }\end{array}$ & \\
\hline \multirow[t]{2}{*}{ Drug-level feedback } & HPTN 082 & $\begin{array}{l}\text { Drug-level feedback counseling was not effective } \\
\text { in increasing PrEP adherence }\end{array}$ & $\begin{array}{l}\text { Drug-level feedback can be costly and logistically } \\
\text { challenging }\end{array}$ \\
\hline & PrEP SMART & Ongoing study & \\
\hline \multirow[t]{2}{*}{ Adherence counseling } & EMPOWER & $\begin{array}{l}\text { GBV screening and counseling acceptable and } \\
\text { feasible }\end{array}$ & $\begin{array}{l}\text { Drop-offs in retention for clinic visits, provider } \\
\text { training and time for counseling delivery }\end{array}$ \\
\hline & PrEP SMART & Ongoing study & \\
\hline \multirow[t]{2}{*}{ Peer groups } & EMPOWER & $\begin{array}{l}\text { Peer based club did not enhance PrEP adherence; } \\
\text { clubs were acceptable, valuable for sharing tips } \\
\text { on managing side effects }\end{array}$ & $\begin{array}{l}\text { AGYW have busy schedules led to poor attendance } \\
\text { of the clubs }\end{array}$ \\
\hline & HPTN 082 & $\begin{array}{l}\text { Monthly adherence clubs acceptable. Approxi- } \\
\text { mately } 70 \% \text { AGYW enrolled in the trial attended } \\
\text { at least one adherence club at three months of } \\
\text { PrEP use }\end{array}$ & \\
\hline Decision support tool & POWER & $\begin{array}{l}\text { AGYW who had access to the tool had } 20 \% \text { con- } \\
\text { tinuation, compared to } 11 \% \text { in the other website, } \\
\text { resulting in } 1.97 \text { times the odds of PrEP continu- } \\
\text { ation at month } 1 \text { than those in usual care }\end{array}$ & $\begin{array}{l}\text { Limited time for completing the decision tool, } \\
\text { smartphone access, phone data }\end{array}$ \\
\hline
\end{tabular}


PrEP users, as well as virtual social support groups. Recent evidence has found that mHealth interventions are a highly

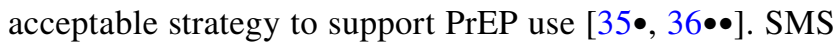
reminder messages could potentially help AGYW develop adherence habits and provide a sense of connection and sup-

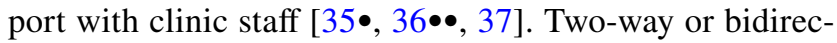
tional SMS messages also enable new PrEP users to communicate with a remote provider, ask PrEP-related questions, and address concerns around pill-taking and side effects that may not have been answered during a clinic visit.

While SMS interventions are acceptable for young women using PrEP and have been found to effectively support ART adherence among women, they have not been found to effectively improve PrEP adherence among AGYW, where adherence is defined using electronic monitoring devices capturing dosing over a prolonged period of time. In a recent trial of SMS reminder messages among Kenyan AGYW, the SMS intervention did not significantly improve PrEP adherence in the context of generally low PrEP use across a 24-month follow-up period [36••]. PrEP demonstration projects have consistently shown a decline in PrEP use through the first 3-6 months after PrEP initiation [9, $10,12,38 \bullet \bullet, 39,40]$, and it is possible that SMS interventions have a limited impact on PrEP use in part because of drop-offs in PrEP adherence (and AGYW needs for PrEP) in both intervention and standard-of-care arms. In addition, participants may experience SMS fatigue over a long study period. Ideally, SMS interventions could be used to support early PrEP habit-formation, adherence, and continuation particularly over the first 3 months of use or during a time when an AGYW is resuming PrEP use after a break, but more research is needed to understand the optimal approach for a youth-friendly, SMS intervention. Cost of these SMS programs will also need to be considered prior to implementing such services if they are found to be effective for AGYW. A recent study evaluating one-way and two-way SMS interventions for ART adherence in Kenya found that one-way SMS costs approximately \$2542 and two-way SMS costs approximately $\$ 3725$ per facility and 115 individuals reached [41]. Personnel and software development comprised the majority of these costs [41]. Other work using SMS for voluntary medical male circumcision in Zimbabwe found that while two-way SMS had a high upfront cost and other costs associated with maintenance, it could successfully reduce clinic visit and outreach costs [41, 42]. Similar budget impact and cost-effectiveness analyses are needed to consider the scalability of SMS PrEP support approaches for AGYW.

In addition to these SMS approaches, PrEP delivery programs could also leverage rapidly evolving mobile phone technologies to create PrEP support interventions as smartphone applications. WhatsApp is a commonly used chat platform among AGYW globally and can facilitate virtual discussions around PrEP pill-taking and HIV prevention behaviors. WhatsApp groups have the potential to overcome logistical barriers of in-person clinic visits and currently the "PrEP SMART" study in South Africa is investigating the effect of a two-way SMS intervention compared with WhatsApp groups on PrEP adherence and persistence among AGYW (clinicaltrials.gov; NCT04038060).

\section{Drug-Level Feedback Interventions}

In early PrEP efficacy trials, young women expressed desires to learn more about levels of PrEP in their bodies to motivate regular pill-taking behavior and instill confidence that PrEP was "working" for them [19, 43]. More recent PrEP studies have sought to estimate the effectiveness of "druglevel feedback counseling", where individuals are counseled about the levels of PrEP detected in their blood or urine, on PrEP adherence and continuation. The HPTN 082 study randomized AGYW in South Africa and Zimbabwe to receive either drug-level feedback counseling or standard counseling and did not detect a significant intervention effect on PrEP adherence, defined using dried blood spot data after a year of

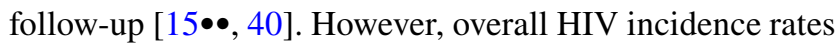
were low in the cohort and high drop-offs in PrEP adherence were observed in both arms. The " $3 \mathrm{P}$ " open-label study (Partners, Perceptions, and Pills) found that drug-level feedback effectively supports PrEP habit formation, although the extent to which it impacted PrEP adherence remains unclear as feedback was combined with PrEP adherence counseling in this program $[44 \bullet \bullet]$. Nonetheless, findings show that drug-level feedback counseling is highly acceptable for

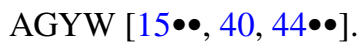

Drug-level feedback counseling is challenging to implement in resource-constrained settings. The $3 \mathrm{P}$ and HPTN 082 studies delivered counseling messages based on PrEP drug levels from dried blood spot (DBS) samples. However, DBS can be expensive to process, can result in logistical challenges and delays in receiving results, and reflects a long window of PrEP pill-taking which does not allow counselors to provide targeted, real-time feedback about recent PrEP use. The "PrEP SMART" study is currently exploring whether a urine point-of-care test is an acceptable means of delivering drug-level feedback counseling to AGYW with more real-time results and lower logistical and cost burden than DBS. Findings from this work, expected in 2022, will inform future drug-level feedback counseling options for young women.

\section{PrEP Adherence Counseling Interventions}

Adherence counseling at PrEP initiation and refill visits is essential for promoting informed PrEP decisions and PrEP continuation and persistence among AGYW. Ongoing PrEP 
studies are exploring individually, tailored adherence counseling, using strategies such as cognitive behavioral therapy, motivational interviewing, and problem-solving to overcome barriers to PrEP use for AGYW (e.g., PrEP SMART). The EMPOWER study assessed the combination of GBV and PrEP adherence counseling as it is hypothesized that GBV experiences may serve as a barrier to PrEP use. Findings from this study showed that GBV screening and counseling was acceptable and feasible for implementation with PrEP counseling [45].

\section{Peer Support Groups}

Adolescence is a critical time of social network development and strengthening and peer support groups provide an important platform for AGYW to share PrEP taking experiences and offer tips and advice. In EMPOWER, peer-based empowerment clubs did not enhance PrEP adherence [46]. However, the qualitative findings show that they are acceptable, enable users to share tips and tricks to overcome side effects, build a social network [47•]. Similarly, in HPTN 082 trial, PrEP adherence clubs were acceptable among AGYW taking PrEP. Approximately $70 \%$ of AGYW enrolled in the trial attended at least one adherence club at 3 months of PrEP use [16]. Implementation of in-person support clubs may be challenging in contexts where there may be lower social cohesion and AGYW can have difficulty attending groups because of busy schedules and competing activities. Current PrEP programs are exploring virtual PrEP adherence groups via WhatsApp as an alternative means to facilitate peer support.

\section{PrEP Decision Support Tools}

PrEP decision tools, delivered via mobile platforms, offer another recent innovation that has shown potential to enhance PrEP uptake and adherence in AGYW. The tools are used to provide PrEP information and risk perception assessment. They are particularly useful in instances where there is limited time to educate patients in busy clinics and limited experience of providers with patient-centered counseling and can overcome provider barriers to delivery, especially in resource-limited settings. They also have the potential to improve PrEP use by addressing knowledge about HIV risk and risk perceptions and can help with PrEP decision-making among AGYW who feel hesitant to initiate discussions about PrEP with providers due to concerns about providers' judgmental attitudes and stigma. Recent evidence on the "MyPrEP" phone-based decision tool found that the tool is associated with increased PrEP persistence. AGYW who had access to the tool had $20 \%$ continuation, compared to $11 \%$ in the other website, resulting in 1.97 times the odds of PrEP continuation at month 1 than those in usual care (conference abstract in process). More studies are underway assessing the effect of PrEP decision tools, integrated with decision support messaging around oral PrEP and injectables.

\section{Community and Health Systems Interventions to Promote Youth-Friendly PrEP Delivery}

Although individual and interpersonal levels are critical to address individual-level barriers to PrEP use, there is also a need for community and health systems-level interventions to promote PrEP use for AGYW. Making PrEP acceptable to a broader community, easily accessible to young people, and delivered in environments that are youth-friendly and welcoming is a priority for programs. Recent research has sought to promote successful PrEP delivery and use among AGYW through: (1) adolescent-friendly PrEP demand creation in communities and clinics; (2) modified PrEP refill and visit schedules; and (3) community-based and de-medicalized PrEP delivery approaches and PrEP delivery integrated with other services for gender-based violence, mental health, and sexual and reproductive health (Table 2).

\section{Adolescent-Friendly PrEP Demand Creation: Best Approaches to Deliver PrEP Information}

PrEP awareness and demand creation among AGYW is key to facilitate uptake and effective use. PrEP messages that are simple and motivate young women to consider their HIV risk while also appealing to their emotions and aspirations may be effective for this population. HIV prevention messages can be delivered through community avenues that are attractive and easily accessible by youth, including radio, television, and social media platforms [48•, 49]. For example, in the "3P" (Partners, Perceptions, and Pills) HIV prevention study conducted among AGYW in South Africa, a 90-s video showcasing youth seeking PrEP from an empowerment lens was highly acceptable and generated excitement among AGYW [50•]. The project found high interest in PrEP among participants who viewed the video.

It is also critical to ensure the broader community in which AGYW are situated is aware of and accepting of young people using PrEP, given that decisions about PrEP uptake and continuation are often motivated by peers, sexual partners, family members, and the community [25]. AGYW are particularly motivated to take PrEP effectively when they hear about it from others like them. HIV treatment studies have found that peer support interventions can successfully improve AGYW engagement in HIV care services including adherence to daily pill-taking for HIV treatment [51]. For a recent PrEP program in Kenya, the use of peer providers to promote PrEP awareness was associated with increased 
Table 2 Summary of current findings on community and health systems interventions to promote youth-friendly PrEP delivery

\begin{tabular}{|c|c|c|c|}
\hline Intervention type & Study & Country & Key findings \\
\hline \multirow[t]{4}{*}{ Adolescent-friendly PrEP demand creation } & $3 \mathrm{P}$ & South Africa & $\begin{array}{l}\text { A PrEP video developed for demand creation was } \\
\text { accepted by AGYW and generated high demand for } \\
\text { PrEP }\end{array}$ \\
\hline & Jilinde Project & Kenya & $\begin{array}{l}\text { Over a 2-year period trained youth peer providers (YPP) } \\
\text { reached 30,713 AGYW with PrEP messages compared } \\
\text { to } 8853 \text { reached by community health volunteers }\end{array}$ \\
\hline & HPTN 082 & South Africa, Zimbabwe & $\begin{array}{l}\text { There was high acceptability for adherence clubs with } \\
70 \% \text { of enrolled AGYW attending at least one adher- } \\
\text { ence club at } 3 \text { months of PrEP use }\end{array}$ \\
\hline & PrIYA & Kenya & $\begin{array}{l}\text { Qualitative interviews with women using PrEP in preg- } \\
\text { nancy identified health providers as facilitators of PrEP } \\
\text { use who could explain PrEP to male partners on behalf } \\
\text { of pregnant women }\end{array}$ \\
\hline Modified PrEP refill and visit schedules & PlusPills & South Africa & $\begin{array}{l}\text { PrEP usage decreased and adherence among adolescents } \\
\text { diminished when visits became less frequent }\end{array}$ \\
\hline $\begin{array}{l}\text { Community-based, de-medicalized, and } \\
\text { integrated PrEP delivery approaches }\end{array}$ & POWER & Kenya, South Africa & $\begin{array}{l}\text { PrEP services for AGYW are offered at family plan- } \\
\text { ning clinics, youth friendly clinics, and mobile youth } \\
\text { friendly clinics with high uptake across these sites }\end{array}$ \\
\hline
\end{tabular}

PrEP uptake among AGYW [52]. However, the role of peers in promoting PrEP continuation has yet to be established. Another peer-based PrEP support approach, "PrEP My Way", is currently under study in Kisumu, Kenya, and uses peers to educate AGYW and deliver PrEP medication (clinicaltrials.gov: NCT04408729). Results are expected in 2023.

Healthcare providers also have an important role in delivering PrEP messaging and supporting PrEP use among AGYW within health facilities. Young PrEP users report increased motivation to adhere to PrEP when providers engage with them in a youth-friendly way, call them to check in on how they are, and send reminder messages about upcoming clinic visits [53•]. In Kenya, young pregnant women taking part in the PrIYA study reported that providers also facilitated their PrEP use by helping to explain PrEP to their male partners and family members [54]. It is necessary that service delivery environments are non-judgmental and supportive for AGYW and prior studies have found that health provider training can effectively address biases and improve quality of care, which could in turn promote care uptake and engagement among young people [21, 55].

\section{Modified PrEP Refill and Visit Schedules}

The World Health Organization recommends that individuals on PrEP return to clinic for a scheduled visit 1 month after PrEP initiation and then receive HIV testing and refills every 3 months thereafter [56]. Countries throughout Africa, where AGYW comprise a disproportionate burden of incident HIV infections, have adopted and implemented this recommendation [28]. However, data suggest that quarterly clinic visits may not work well for some AGYW. A recent study conducted among AGYW in South Africa observed a reduction in PrEP continuation when medication refill visits changed from monthly to quarterly [12]. This finding suggests that AGYW may benefit from more frequent clinic visits to address dynamic changes in their routines and HIV prevention needs. For those who cannot physically come to a clinic regularly, interactions with healthcare providers could take place by phone, text message, or WhatsApp and the COVID-19 pandemic has provided a unique opportunity to explore the acceptability and feasibility of telemedicine

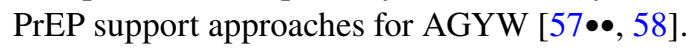

\section{Community-based, De-medicalized, and Integrated PrEP Delivery Approaches}

Siloes in the healthcare delivery system can prevent AGYW from receiving PrEP alongside other medical services. AGYW commonly present to public facilities to receive sexual and reproductive health services [29••]. Therefore, establishing PrEP delivery within antenatal, postnatal, and family planning clinics has the potential to increase PrEP

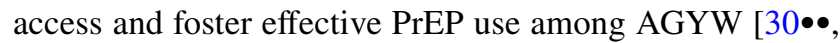
$59 \bullet, 60]$. Doing so also promotes a holistic approach to addressing the healthcare needs of AGYW. Ongoing work is also exploring approaches to integrate PrEP delivery with sexually transmitted infection testing and treatment and mental health service delivery.

Delivery of PrEP services outside of clinical settings is also a promising approach to foster effective PrEP use among young women. Recent PrEP projects have been exploring the use of mobile vans and community-based venues for PrEP delivery. For example, the ongoing Prevention 
Options for Women Evaluation Research (POWER) study is offering PrEP services out of mobile, youth-friendly clinics in South Africa and has demonstrated high PrEP uptake in this setting (clinicaltrials.gov; NCT03490058). Similarly, the DREAMS initiative, which operates in several African countries, is currently providing PrEP in a package of comprehensive, integrated services out of safe spaces within communities. Community pharmacies delivery healthcare services in a relatively anonymous and convenient manner and may also be attractive venues for PrEP delivery to AGYW. Pharmacy-based PrEP delivery programs have successfully improved PrEP accessibility for at-risk individuals in developed countries and could be adapted for the African context as well $[61 \bullet, 62]$. We expect results on the impact of these integrated, de-medicalized PrEP delivery approaches over the next few years. The studies highlighted here have been predominantly conducted with AGYW in South Africa and Kenya and we also look forward to the broader application of these PrEP delivery approaches to AGYW in other settings.

\section{Conclusion}

Recent advances in oral PrEP delivery for AGYW include an exciting variety of individual-, interpersonal-, health systems-, and community-level interventions, being tested and rolled out in the context of redefined metrics for success. While the interventions highlighted here have shown promise as acceptable and feasible for delivery with AGYW, limited data on the effectiveness of these interventions in promoting PrEP uptake, prevention-effective adherence, and continuation during periods of HIV risk are currently available. The research landscape is rapidly evolving and we look forward to forthcoming findings from the next generation of PrEP support interventions and long-acting HIV prevention options for AGYW [63•]. Moreover, these approaches are not one-size-fits-all, for the individual, the health system, or the cultural context, and choice of these intervention approaches will depend on the population's needs and setting. Tailored, multi-level PrEP support approaches are critical to maximize the public health benefit of PrEP and reduce the burden of HIV among AGYW in HIV endemic settings.

\section{References}

Papers of particular interest, published recently, have been highlighted as:

- Of importance

• Of major importance
1. Baeten JM, Palanee-Phillips T, Brown ER, Schwartz K, SotoTorres LE, Govender V, et al. Use of a vaginal ring containing dapivirine for HIV-1 prevention in women. N Engl J Med. 2016;375(22):2121-32.

2. Evidence for Contraceptive Options and HIV Outcomes (ECHO) Trial Consortium. HIV incidence among women using intramuscular depot medroxyprogesterone acetate, a copper intrauterine device, or a levonorgestrel implant for contraception: a randomised, multicentre, open-label trial. Lancet. 2019 27;394(10195):303-13. This article provides data from the first randomized controlled assessing the effect of hormonal and non-hormonal contraceptive options on HIV risk. The authors did not find a substantial difference in HIV risk by contraceptive method, indicating the importance of contraceptive method choice for young women in HIV endemic settings.

3. UNAIDS. Trend in new infections: Eastern, Southern, Western, Central Africa by Sex. [Internet]. 2018 [cited 2019 Jul 31]. Available from: aidsinfo.unaids.org

4. Baggaley R, Armstrong A, Dodd Z, Ngoksin E, Krug A. Young key populations and HIV: a special emphasis and consideration in the new WHO Consolidated Guidelines on HIV Prevention, Diagnosis, Treatment and Care for Key Populations. J Int AIDS Soc. 2015;18(2 Suppl 1):19438.

5. Grant RM, Lama JR, Anderson PL, McMahan V, Liu AY, Vargas L, et al. Preexposure chemoprophylaxis for HIV prevention in men who have sex with men. $\mathrm{N}$ Engl J Med. 2010;363(27):2587-99.

6. Baeten JM, Donnell D, Ndase P, Mugo NR, Campbell JD, Wangisi J, et al. Antiretroviral prophylaxis for HIV prevention in heterosexual men and women. N Engl J Med. 2012;367(5):399-410.

7. Choopanya K, Martin M, Suntharasamai P, Sangkum U, Mock PA, Leethochawalit M, et al. Antiretroviral prophylaxis for HIV infection in injecting drug users in Bangkok, Thailand (the Bangkok Tenofovir Study): a randomised, double-blind, placebo-controlled phase 3 trial. Lancet. 2013;381(9883):2083-90.

8. Thigpen MC, Kebaabetswe PM, Paxton LA, Smith DK, Rose CE, Segolodi TM, et al. Antiretroviral preexposure prophylaxis for heterosexual HIV transmission in Botswana. N Engl J Med. 2012;367(5):423-34.

9. Bekker LG, Roux S, Sebastien E, Yola N, Amico KR, Hughes JP, et al. Daily and non-daily pre-exposure prophylaxis in African women (HPTN 067/ADAPT Cape Town Trial): a randomised, open-label, phase 2 trial. Lancet HIV. 2017;5(2):e68-78.

10. Hosek SG, Rudy B, Landovitz R, Kapogiannis B, Siberry G, Rutledge B, et al. An HIV preexposure prophylaxis demonstration project and safety study for young MSM. J Acquir Immune Defic Syndr. 2017 01;74(1):21-9.

11.•• Grinsztejn B, Hoagland B, Moreira RI, Kallas EG, Madruga JV, Goulart S, et al. Retention, engagement, and adherence to pre-exposure prophylaxis for men who have sex with men and transgender women in PrEP Brasil: 48 week results of a demonstration study. Lancet HIV. 2018;5(3):e136-45. This openlabel PrEP demonstration project in Brazil reported that 450 individuals initiated PrEP in a real-world delivery setting through public healthcare clinics and found very high retention rates $(83 \%)$ on PrEP by week 48 . In addition, $74 \%$ of participants had high levels of PrEP drug concentrations indicating approximately four doses per week at the week 48 visit.

12. Gill K, Dietrich J, Gray G, Pidwell T, Kayamba E, Bennie, T, Myer L, Johnson L, Spiegel H, Slack C, Elharrar V, Strode A, Rooney J, Bekker LG. Pluspills: an open label, safety and feasibility study of oral pre-exposure prophylaxis (PrEP) in 15-19 year old adolescents in two sites in South Africa. Abstract \#TUAC0207LB. Oral presentation at the 9th International AIDS 
Society Conference (IAS) on HIV Science; July 23-26, 2017; Paris, France.

13. Amico KR, Wallace M, Bekker LG, Roux S, Atujuna M, Sebastian E, et al. Experiences with HPTN 067/ADAPT study-provided open-label PrEP among women in Cape Town: facilitators and barriers within a mutuality framework. AIDS Behav. 2017;21(5):1361-75.

14. Allison BA, Widman L, Stewart JL, Evans R, Perry M. Adherence to pre-exposure prophylaxis in adolescents and young adults: a systematic review and meta-analysis. J Adolesc Health. 2021;S1054-139X(21)00169-5. This meta-analysis provides data on PrEP adherence across PrEP trials in a number of settings and populations. The findings reveal that adolescent girls and young women had lower adherence than other young key populations (about $46 \%$ of adolescent girls and young women adhered to PrEP across PrEP studies).

15.• Celum C, Hosek S, Tsholwana M, Kassim S, Mukaka S, Dye BJ, et al. PrEP uptake, persistence, adherence, and effect of retrospective drug level feedback on PrEP adherence among young women in southern Africa: results from HPTN 082, a randomized controlled trial. PLoS Med. 2021;18(6):e1003670. This manuscript presents findings from the HPTN 082 trial conducted with adolescent girls and young women in South Africa and Zimbabwe. The study did not find that drug-level feedback counseling improved PrEP adherence in this population, but PrEP continuation and HIV incidence were low overall.

16. Essien T, Mhlanga N, Hinson K, Mzizi P, Johnson A, Chasakara $\mathrm{C}$, et al. Engaging adolescent girls and young women in HPTN 082: lessons learned from an oral PrEP study. Poster presentation at the $10^{\text {th }}$ International AIDS Society (IAS) Conference on HIV Science; July 21-24, 2019; Mexico City, Mexico.

17. Cabral A, M Baeten J, Ngure K, Velloza J, Odoyo J, E Haberer $\mathrm{J}$, et al. Intimate partner violence and self-reported pre-exposure prophylaxis interruptions among HIV-negative partners in HIV serodiscordant couples in Kenya and Uganda. J Acquir Immune Defic Syndr. 2018;77(2):154-9.

18. Velloza J, Khoza N, Scorgie F, Chitukuta M, Mutero P, Mutiti K, et al. The influence of HIV-related stigma on PrEP disclosure and adherence among adolescent girls and young women in HPTN 082: a qualitative study. J Int AIDS Soc. 2020;23(3):e25463. This manuscript provides descriptive data on experiences of PrEP-related stigma, disclosure, and PrEP use among adolescent girls and young women in South Africa and Zimbabwe. Stigma was a barrier to PrEP disclosure shortly after PrEP initiation but disclosure skills could also be used to combat stigma over time.

19. van der Straten A, Stadler J, Montgomery E, Hartmann M, Magazi B, Mathebula F, et al. Women's experiences with oral and vaginal pre-exposure prophylaxis: the VOICE-C qualitative study in Johannesburg, South Africa. PloS One. 2014;9(2):e89118.

20. Velloza J, Hosek S, Donnell D, Anderson PL, Chirenje M, Mgodi N, et al. Assessing longitudinal patterns of depressive symptoms and the influence of symptom trajectories on HIV preexposure prophylaxis adherence among adolescent girls in the HPTN 082 randomized controlled trial. J Int AIDS Soc. 2021;24 Suppl 2:e25731.

21. Pilgrim N, Jani N, Mathur S, Kahabuka C, Saria V, Makyao $\mathrm{N}$, et al. Provider perspectives on PrEP for adolescent girls and young women in Tanzania: the role of provider biases and quality of care. PloS One. 2018;13(4):e0196280.

22.•• Dunbar MS, Kripke K, Haberer J, Castor D, Dalal S, Mukoma $\mathrm{W}$, et al. Understanding and measuring uptake and coverage of oral pre-exposure prophylaxis delivery among adolescent girls and young women in sub-Saharan Africa. Sex Health. 2018;15(6):513-21. This manuscript describes an intervention-centric cascade for defining metrics around PrEP uptake and coverage for adolescent girls and young women (AGYW). It also emphasizes the need to reframe definitions of "low uptake" to focus on delivering oral PrEP to AGYW during periods of HIV risk when they most need PrEP.

23. Haberer JE. Current concepts for PrEP adherence in the PrEP revolution: from clinical trials to routine practice. Curr Opin HIV AIDS. 2016;11(1):10-7.

24. Celum CL, Delany-Moretlwe S, Baeten JM, van der Straten A, Hosek S, Bukusi EA, et al. HIV pre-exposure prophylaxis for adolescent girls and young women in Africa: from efficacy trials to delivery. J Int AIDS Soc. 2019;22 Suppl 4:e25298.

25. Haberer JE, Mugo N, Baeten JM, Pyra M, Bukusi E, Bekker LG. PrEP as a lifestyle and investment for adolescent girls and young women in Sub-Saharan Africa. J Int Assoc Provid AIDS Care. 2019;18:2325958219831011.

26. Abaasa A, Hendrix C, Gandhi M, Anderson P, Kamali A, Kibengo $\mathrm{F}$, et al. Utility of different adherence measures for PrEP: patterns and incremental value. AIDS Behav. 2018;22(4):1165-73. This manuscript found that selfreported PrEP adherence metrics are of limited usefulness alone but their utility can be improved when combined with biomarker measures of PrEP adherence.

27. Musinguzi N, Muganzi CD, Boum Y, Ronald A, Marzinke MA, Hendrix CW, et al. Comparison of subjective and objective adherence measures for preexposure prophylaxis against HIV infection among serodiscordant couples in East Africa. AIDS. 2016;30(7):1121-9.

28. Bekker L-G, Rebe K, Venter F, Maartens G, Moorhouse M, Conradie F, et al. Southern African guidelines on the safe use of pre-exposure prophylaxis in persons at risk of acquiring HIV-1 infection. South Afr J Hiv Med. 2016;17(1).

29.•• Ongolly FK, Dolla A, Ngure K, Irungu EM, Odoyo J, Wamoni E, et al. "I just decided to stop:" understanding PrEP discontinuation among individuals initiating PrEP in HIV care centers in Kenya. J Acquir Immune Defic Syndr. 2021;87(1):e150-8. This manuscript describes experiences of PrEP discontinuation, including reasons for intentional discontinuation such as reduced HIV risk, in the context of a large PrEP roll-out program in Kenya.

30.• Mugwanya KK, Pintye J, Kinuthia J, Abuna F, Lagat H, Begnel $\mathrm{ER}$, et al. Integrating preexposure prophylaxis delivery in routine family planning clinics: a feasibility programmatic evaluation in Kenya. PLoS Med. 2019;16(9):e1002885. This manuscript presents findings from a real-world PrEP implementation program, integrated with family planning service delivery, in Kenya and found that integrated servcies were feasible and acceptable for young women.

31. Gilbert HN, Wyatt MA, Pisarski EE, Muwonge TR, Heffron R, Katabira ET, et al. PrEP discontinuation and prevention-effective adherence: experiences of PrEP users in Ugandan HIV serodiscordant couples. JAIDS. 2019 Nov 1;82(3):265. This manuscript describes qualitative experiences of PrEP continuation and discontinuation among HIV sero-discordant couples. The authors found that participants saw discontinuation as a loss of HIV protection even when partners living with HIV were virally suppressed, citing challenges for real-world counseling about effective PrEP use.

32. Haberer JE, Bangsberg DR, Baeten JM, Curran K, Koechlin F, Amico KR, et al. Defining success with HIV pre-exposure prophylaxis: a prevention-effective adherence paradigm. AIDS. 2015;29(11):1277-85.

33. Haberer JE, Kidoguchi L, Heffron R, Mugo N, Bukusi E, Katabira $\mathrm{E}$, et al. Alignment of adherence and risk for HIV acquisition in a demonstration project of pre-exposure prophylaxis 
among HIV serodiscordant couples in Kenya and Uganda: a prospective analysis of prevention-effective adherence. J Int AIDS Soc. 2017;20(1).

34. Scorgie F, Khoza N, Delany-Moretlwe S, Velloza J, Mangxilana N, Atujuna M, et al. Narrative sexual histories and perceptions of HIV risk among young women taking PrEP in southern Africa: findings from a novel participatory method. Soc Sci Med. 2020;270:113600. This manuscript describes findings from a novel qualitative approach to capture information on HIV risk perceptions and desires for PrEP related to sexual partnerships. Adolescent girls and young women were most concerned about HIV in relationships where they felt a lack of trust and agency.

35. Muwonge TR, Ngure K, Katabira E, Mugo N, Kimemia G, Burns BFO, et al. Short message service (SMS) surveys assessing pre-exposure prophylaxis (PrEP) adherence and sexual behavior are highly acceptable among HIV-uninfected members of serodiscordant couples in East Africa: a mixed methods study. AIDS Behav. 2019;23(5):1267-1276. The authors assessed the acceptability and feasibility of using SMS surveys to collect sexual behavior data and found that most participants reported the SMS messages were easy to use and preferable to clinic questionnaires.

36.• Haberer JE, Bukusi EA, Mugo NR, Pyra M, Kiptinness C, Oware $\mathrm{K}$, et al. Effect of SMS reminders on PrEP adherence in young Kenyan women (MPYA study): a randomised controlled trial. Lancet HIV. 2021;8(3):e130-7. This manuscript presents findings from the MPYA randomized trial of an SMS-based PrEP adherence support intervention. They found that SMS reminders were ineffective in promoting PrEP adherence among young Kenyan women.

37. Georgette N, Siedner MJ, Zanoni B, Sibaya T, Petty CR, Carpenter S, et al. The Acceptability and Perceived Usefulness of a Weekly Clinical SMS Program to Promote HIV Antiretroviral Medication Adherence in KwaZulu-Natal. South Africa AIDS Behav. 2016;20(11):2629-38.

38.• Rousseau-Jemwa E, Bekker L-G, Bukusi E, Delaney-Moretlwe S, Omollo V, Travill D, et al. Early persistence of HIV pre-exposure prophylaxis (PrEP) in African adolescent girls and young women (AGYW) from Kenya and South Africa. AIDS Res Hum Retroviruses. 2018;34:68-68. This manuscript reviews findings from PrEP demonstration projects for adolescent girls and young women and found that PrEP is feasible for implementation in sexual and reproductive health service delivery models.

39. Travill D, Bekker L-G, Delaney-Moretlwe S, Bukusi E, Imrie J, Rousseau-Jemwa E, et al. Sexual behavior and PrEP uptake among young African women in a demonstration project about PrEP delivery. Abstract \#WEPEC219. Oral Presentation at the $9^{\text {th }}$ International AIDS Society Conference (IAS); July 23-27, 2018; Amsterdam, the Netherlands.

40. Celum C, Mgodi N, Bekker LG, Hosek S, Donnell D, Anderson PL, Dye BJ, Pathak S, Agyei Y, Fogel JM, Marzinke MA, Makgamathe K, Kassim S, Mukaka S, Noble H, Adeyeye A, Delany-Moretlwe $\mathrm{S}$, on behalf of the HPTN 082 study team. PrEP adherence and effect of drug level feedback among young African women in HPTN 082. Abstract \#TUAC0301. Oral presentation at the International AIDS Society Conference (IAS); July 21-24, 2019; Mexico City, Mexico.

41. Chen Y, Ronen K, Matemo D, Unger JA, Kinuthia J, JohnStewart G, Levin C. An interactive text messaging intervention to improve adherence to Option B+ prevention of mother-tochild HIV transmission in Kenya: cost analysis. JMIR mHealth uHealth. 2020;8(10):e18351.

42. Babigumira JB, Barnhart S, Mendelsohn JM, Murenje V, Tshimanga M, Mauhy C, Holeman I, Xaba S, Holec
MM, Makunike-Chikwinya B, Feldacker C. PloS One. 2020;15(9):e0239915.

43. van der Straten A, Stadler J, Luecke E, Laborde N, Hartmann M, Montgomery ET. Perspectives on use of oral and vaginal antiretrovirals for HIV prevention: the VOICE-C qualitative study in Johannesburg, South Africa. J Int AIDS Soc. 2014;17(3Suppl 2).

44.• Celum CL, Gill K, Morton JF, Stein G, Myers L, Thomas $\mathrm{KK}$, et al. Incentives conditioned on tenofovir levels to support PrEP adherence among young South African women: a randomized trial. J Int AIDS Soc. 2020;23(11):e25636. This manuscript presents findings from the $3 P$ study, which provided incentives to promote PrEP adherence among adolescent girls and young women in South Africa. The study did not find a significant effect of the interventions but PrEP adherence and persistence was high in the cohort.

45. Colombini M, Mayhew SH, Mutemwa R, Kivunaga J, Ndwiga C, Team TI. Perceptions and experiences of integrated service delivery among women living with HIV attending reproductive health services in Kenya: a mixed methods study. AIDS Behav. 2016;20(9):2130-40.

46. Delaney-Moretlwe S, Chersich M, Harvey S, Stangl A, Baron D, Columbini M, et al. Empowerment clubs did not increase PrEP continuation among adolescent girls and young women in South Africa and Tanzania - results from the EMPOWER randomised trial. Abstract \# FRAE0108LB. Oral presentation at the $9^{\text {th }}$ International AIDS Society Conference (IAS); July 23-27, 2018; Amsterdam, the Netherlands.

47. Baron D, Scorgie F, Ramskin L, Khoza N, Schutzman J, Stangl A, et al. "You talk about problems until you feel free": South African adolescent girls' and young women's narratives on the value of HIV prevention peer support clubs. BMC Public Health. 2020;20(1):1016. This manuscript presents qualitative data from the EMPOWER study, providing insights into the benefits of support groups around gender-based violence and PrEP use.

48. Masyuko S, Mukui I, Njathi O, Kimani M, Oluoch P, Wamicwe J, et al. Pre-exposure prophylaxis rollout in a national public sector program: the Kenyan case study. Sex Health. 2018;15(6):578-86. This manuscript describes the Kenyan experience of rolling out PrEP for high risk populations, including adolescent girls and young women, and could serve as a model for other settings.

49. Coombs A, Gold E. Generating Demand for PrEP: a desk review. Arlington, VA, USA; 2019. (Strengthening High Impact Interventions for an AIDS-Free Generation Project).

50.• Morton JF, Myers L, Gill K, Bekker L-G, Stein G, Thomas $\mathrm{KK}$, et al. Evaluation of a behavior-centered design strategy for creating demand for oral PrEP among young women in Cape Town, South Africa. Gates Open Res. 2020;4:29. This manuscript describes the use of a behavior-centered design (BCD) approach to develop a PrEP social marketing campaign for adolescent girls and young women in South Africa. Participants discussed desires for a positively framed, empowering PrEP demand creation video.

51. Mark D, Hrapcak S, Ameyan W, Lovich R, Ronan A, Schmitz K, et al. Peer support for adolescents and young people living with HIV in sub-Saharan Africa: emerging insights and a methodological agenda. Curr HIV/AIDS Rep. 2019;16(6):467-74.

52. Agunda P, Mutisya E, Musau A, Odera C, Maikweki L, Wango B, et al. Increasing Uptake of Oral Pre-Exposure Prophylaxis among Adolescent Girls and Young Women through Youth Peer Providers in Migori County, Kenya. Abstract \#44. Oral presentation at the International Workshop on HIV and Adolescence; 2019; Nairobi, Kenya. 
53.• Wood S, Gross R, Shea JA, Bauermeister JA, Franklin J, Petsis D, et al. Barriers and facilitators of PrEP adherence for young men and transgender women of color. AIDS Behav. 2019;23(10):2719-29. This manuscript describes findings from a qualitative study on barriers and facilitators for PrEP use among young men who have sex with men and transgender women. Barriers included stigma, health systems inaccessibility, side effects, competing stressors, and low HIV risk perception.

54. Pintye J, Beima-Sofie KM, Makabong'O PA, Njoroge A, Trinidad SB, Heffron RA, et al. HIV-uninfected Kenyan adolescent and young women share perspectives on using pre-exposure prophylaxis during pregnancy. AIDS Patient Care STDs. 2018;32(12):538-44.

55. Wechsberg W, Ahmed K, Ndirangu J, Browne FA, Bonner C, Speizer I, et al. Can we end HIV among adolescent girls and young women in South Africa? Key challenges with PrEP. Abstract \#4042.0. Oral presentation at the American Public Health Association Annual Meeting; November 2-6, 2019; Philadephia, PA, USA.

56. WHO implementation tool for pre-exposure prophylaxis (PrEP) of HIV infection. Module 12: Adolescents and young adults. Geneva, Switzerland: World Health Organization; 2018. Report No.: License: CC BY-NC-SA 3.0 IGO.

57.• Hill BJ, Anderson B, Lock L. COVID-19 Pandemic, Preexposure Prophylaxis (PrEP) care, and HIV/STI testing among patients receiving care in three HIV epidemic priority states. AIDS Behav. 2021;25(5):1361-5. This manuscript provides data on the use of a telehealth/telePrEP approach to promote PrEP prescribing during the COVID pandemic.

58. Alwang'a H. DREAMS goes digital: using virtual platforms to maintain PrEP access among adolescent girls and young women in Kenya. Presentation at the $23^{\text {rd }}$ International AIDS Conference; July 6-10, 2020; Virtual.
59.• Maseko B, Hill LM, Phanga T, Bhushan N, Vansia D, Kamtsendero L, et al. Perceptions of and interest in HIV pre-exposure prophylaxis use among adolescent girls and young women in Lilongwe, Malawi. PloS One. 2020;15(1):e0226062. This manuscript describes facilitators and barriers to PrEP delivery in Malawi. Adolescent girls and young women reported preferences that PrEP is delivered in youth-friendly sections of health centers with youth-friendly counseling messages.

60. Pintye J, Kinuthia J, Roberts DA, Wagner AD, Mugwanya K, Abuna F, et al. Brief report: integration of PrEP services into routine antenatal and postnatal care: experiences from an implementation program in Western Kenya. J Acquir Immune Defic Syndr. 2018;79(5):590-5.

61.• Ortblad KF, Mogere P, Roche S, Kamolloh K, Odoyo J, Irungu E, et al. Design of a care pathway for pharmacy-based PrEP delivery in Kenya: results from a collaborative stakeholder consultation. BMC Health Serv Res. 2020;20(1):1034. The authors describe the development of a pharmacy-based PrEP delivery model, where stakeholders participated in workshops to describe possible challenges with pharmacy-based PrEP delivery and potential implementation approaches.

62. Ortblad KF, Mogere P, Bukusi E, Ngure K, Baeten JM. Pharmacy delivery to expand the reach of PrEP in Africa. J Int AIDS Soc. 2020;23(9):e25619.

63. Coelho LE, Torres TS, Veloso VG, Landovitz RJ, Grinsztejn B. Pre-exposure prophylaxis 2.0: new drugs and technologies in the pipeline. Lancet HIV. 2019;6(11):e788-99.

Publisher's Note Springer Nature remains neutral with regard to jurisdictional claims in published maps and institutional affiliations. 